\title{
Simplified time-domain simulation of detailed long-term dynamic models
}

\author{
Davide Fabozzi Thierry Van Cutsem, Fellow, IEEE
}

\begin{abstract}
Time-domain simulation of power system long-term dynamics involves the solution of large sparse systems of nonlinear stiff differential-algebraic equations. Simulation tools have traditionally focused on the accuracy of the solution and, in spite of many algorithmic improvements, time simulations still require a significant computational effort. In some applications, however, it is sufficient to have an approximate system response of the detailed model. The paper revisits the merits of the Backward Euler method and proposes a strategy to control its step size, with the objective of filtering out fast stable oscillations and focusing on the aperiodic behaviour of the system. The proposed method is compared to detailed simulation as well as to the quasi-steadystate approximation. Illustrative examples are given on a small but representative system, subject to long-term voltage instability.
\end{abstract}

Index Terms-long-term dynamics, stiff decay property, backward Euler method, quasi-steady-state approximation, long-term voltage instability

\section{INTRODUCTION}

$\mathbf{I}$ $\mathrm{N}$ stability studies, the "full" dynamic model of a power system takes on the form:

$$
\begin{aligned}
\mathbf{0} & =\mathbf{g}(\mathbf{x}, \mathbf{y}, \mathbf{z}) \\
\dot{\mathbf{x}} & =\mathbf{f}(\mathbf{x}, \mathbf{y}, \mathbf{z}) \\
\mathbf{z}\left(t_{k}^{+}\right) & =\mathbf{h}\left(\mathbf{x}, \mathbf{y}, \mathbf{z}\left(t_{k}^{-}\right)\right)
\end{aligned}
$$

Behind these simple equations, is hidden a large set of nonlinear, stiff, sparse, differential-algebraic, continuous-discrete time equations [1], [2], [3], [4].

The Algebraic Equations (AEs) (1) deal with the network, whose response is assumed instantaneous under the quasi sinusoidal (or phasor) approximation. The rectangular components of bus voltages are grouped into $\mathbf{y}$.

The Ordinary Differential Equations (ODEs) (2) relate to a wide variety of phenomena and controls ranging from the short-term dynamics of power plants, static var compensators, induction motors, etc., to the long-term dynamics of secondary frequency control, load self restoration, etc. Vector $\mathrm{x}$ includes the corresponding state variables, such as rotor angles, flux linkages, motor slips, controller state variables, etc.

The discrete-time equations (3) capture the discrete controls and protections that act on the system, ranging from the fast switching of shunt compensation to long-term controls such as generator setpoints, load tap changers, overexcitation limiters,

D. Fabozzi (fabozzi@montefiore.ulg.ac.be) is Ph.D. student at the Dept. of Electrical Engineering and Computer Science of the University of Liège, Sart Tilman B37, B-4000 Liège, Belgium.

T. Van Cutsem (t.vancutsem@ulg.ac.be) is a research director of FNRS (Fund for Scientific Research) and adjunct professor at the same department. etc. The corresponding (shunt susceptance, transformer ratio, etc.) variables are grouped into $\mathbf{z}$, which undergoes step changes from $\mathbf{z}\left(t_{k}^{-}\right)$to $\mathbf{z}\left(t_{k}^{+}\right)$at some instant $t_{k}$ [4].

A great number of numerical integration methods has been proposed to transform the ODEs (2) into AEs at each time step of a simulation [5], [6]. Among them, variable-step size algorithms have been also implemented to speed up the computation [6]. As the power systems community technological paradigm for integration has traditionally focused on the accuracy of the solution, integration methods for timedomain simulation with increasing accuracy (and growing complexity) have been proposed [7], [1], [2], [8], [9], [10], [11]. The trapezoidal method has been soon recommended [1] and is widely used. In spite of these improvements, running detailed simulations of the full model still requires a significant computational effort. This effort may be prohibitive for realtime applications, such as dynamic security assessment, for instance.

On the other hand, this high accuracy is not needed in some applications. For instance, when checking long-term voltage instability, the electromechanical oscillations are generally of marginal interest, as long as no generator loses synchronism.

In the search for simplified but efficient time-domain simulation, the full model (1-3) became a target for simplification. One proven technique is the Quasi-Steady-State (QSS) approximation, which is based on time decomposition [4], [12]. It consists in decomposing $\mathbf{x}$ into a fast component $\mathbf{x}_{f}$ and a slow component $\mathbf{x}_{s}$, considering the fast part as infinitely fast and replacing the corresponding ODEs by AEs. Thus, the full model is simplified into:

$$
\begin{aligned}
\mathbf{0} & =\mathbf{g}\left(\mathbf{x}_{f}, \mathbf{x}_{s}, \mathbf{y}, \mathbf{z}\right) \\
\mathbf{0} & =\mathbf{f}_{f}\left(\mathbf{x}_{f}, \mathbf{x}_{s}, \mathbf{y}, \mathbf{z}\right) \\
\dot{\mathbf{x}}_{s} & =\mathbf{f}_{s}\left(\mathbf{x}_{f}, \mathbf{x}_{s}, \mathbf{y}, \mathbf{z}\right) \\
\mathbf{z}\left(t_{k}^{+}\right) & =\mathbf{h}\left(\mathbf{x}_{f}, \mathbf{x}_{s}, \mathbf{y}, \mathbf{z}\left(t_{k}^{-}\right)\right)
\end{aligned}
$$

in which $\mathbf{f}$ has been decomposed into $\mathbf{f}_{f}$ and $\mathbf{f}_{s}$, corresponding to the fast and slow parts, respectively. Instead of using the equations (5) stemming from the detailed model, an ad hoc "reduced" model can be used, as detailed in [4], [12], [13].

The QSS approximation proved to be a good and computationally efficient alternative to full time simulation of a full model; however, the very QSS approximation has limits:

- a long-term degradation of system operating conditions may result in motor stalling or loss of synchronism [4]. In this case, a fast instability of the full model (1-3) appears as an early singularity of the QSS model (4-7) that gives little information about the nature of the problem; 
- following a large disturbance, the system may become unstable in the short-term period and, hence, even not enter the long-term period. Detecting this situation requires to couple detailed and QSS simulations [13];

- a more accurate simulation of long-term dynamics may require to retain some of the fast dynamics [12]. Automatic model simplification methods are to be developed.

Thus, a trade-off has to be found between solution accuracy and completeness, on one hand, and computational efficiency, on the other hand.

This paper presents a new approach for simplified time domain simulation of electric power systems, a compromise between the lengthy simulation of a full model and the fast simulation of a very simplified model. We believe that, while the whole scope of the phenomena to be observed in a power system can only be tracked by means of a full simulation on a full model, there is a range of phenomena that can be covered by a simplified simulation at a reasonable computational cost. Thus, we advocate a paradigm shift from accuracy towards computational efficiency.

The approach exploits the stiff decay property and the simplicity of the backward Euler method to obtain approximate solutions of the full model, without a priori simplification of the latter as in the QSS approach. Besides efficiency, the objectives of the proposed solver are to: (a) ignore fast, hopefully stable, oscillations of lower concern (typically electromechanical inter-machine oscillations), but (b) track fast aperiodic changes that may bring the system to unacceptable operating conditions, and (c) deal efficiently with the numerous discrete events (3).

This paper is organized as follows. Some properties of integration methods are revisited in Section II, while the proposed approach is presented in Section III. A small test system is used in Section IV to illustrate the proposed scheme and compare it to full and QSS simulations. The conclusion in Section V closes the paper.

\section{SHORT REVIEW OF INTEGRATION METHODS}

Given an ODE of the type:

$$
\dot{x}=f(x)
$$

and considering a discretization of the simulation interval in time steps of length $h$, the Trapezoidal Method (TM) computes an approximation of the real solution according to:

$$
x_{n}=x_{n-1}+\frac{h}{2} \dot{x}_{n-1}+\frac{h}{2} \dot{x}_{n}
$$

while the Backward Euler Method (BEM) uses:

$$
x_{n}=x_{n-1}+h \dot{x}_{n}
$$

\section{A. A-Stability}

Consider the simple scalar ODE, often referred to as "test equation":

$$
\dot{x}=\lambda x
$$

where $\lambda$ is a complex number. The region of absolute stability of a given integration method is the region of the $h \lambda$ plane

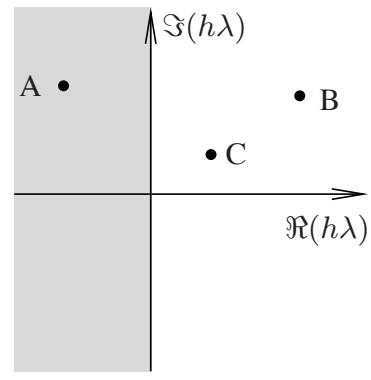

TM

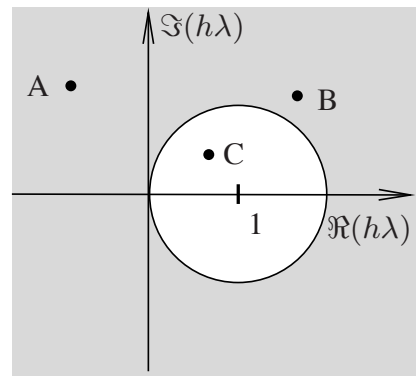

BEM
Fig. 1. Absolute stability regions of the TM and BEM methods

such that applying the method to (11), with $h \lambda$ from within this region, yields a solution satisfying the absolute stability requirement:

$$
\left|x_{n}\right|<\left|x_{n-1}\right|
$$

These regions are shown shaded in Fig. 1, for the TM and BEM methods, respectively [5].

It is desirable that, whenever the exact solution is stable, so is the simulated one. This property is called A-Stability [5] and holds when the region of absolute stability of the method contains the entire left half plane $(h \Re(\lambda)<0)$. Thus both TM and BEM are A-stable.

Thus, for point A in Fig. 1, where $\Re(\lambda)<0$ (and hence $h \Re(\lambda)<0$ ), both TM and BEM produce stable responses. For point $\mathrm{B}$, where the system is unstable, TM produces an unstable response while BEM still gives a stable one. This drawback is referred to as hyper-stability [9]. For point $\mathrm{C}$, the responses of both methods will be unstable.

\section{B. Stiff decay}

As far as computational efficiency is concerned, the possibility of taking large steps is a very important feature. By taking large integration steps, some fast oscillations of lower concern will be ignored. However, in order to accommodate a step size comparable or larger than the period of these oscillations, the integration method must be numerically robust. In this context, the stiff decay property plays a crucial role [5].

Consider the test equation modified as follows:

$$
\dot{x}=\lambda(x-g(t))
$$

where $g(t)$ is a bounded function. An integration method has stiff decay if, for a given $t_{n}>0$ :

$$
\left|x_{n}-g\left(t_{n}\right)\right| \rightarrow 0 \quad \text { as } \quad h \Re(\lambda) \rightarrow-\infty
$$

When TM is applied to (13), it is easily shown that:

$$
x_{n}-g\left(t_{n}\right)=a_{f}\left(x_{n-1}-g\left(t_{n}\right)\right) \quad \text { with } \quad a_{f}=\frac{2+h \lambda}{2-h \lambda}
$$

while with BEM, one has:

$$
x_{n}-g\left(t_{n}\right)=a_{f}\left(x_{n-1}-g\left(t_{n}\right)\right) \quad \text { with } \quad a_{f}=\frac{1}{1-h \lambda}
$$

where $a_{f}$ is often called amplification factor. When $h_{n} \Re(\lambda)$ tends to $-\infty, a_{f}$ tends to -1 for TM and to 0 for BEM. Hence, according to (14), TM does not have stiff decay while BEM has. 


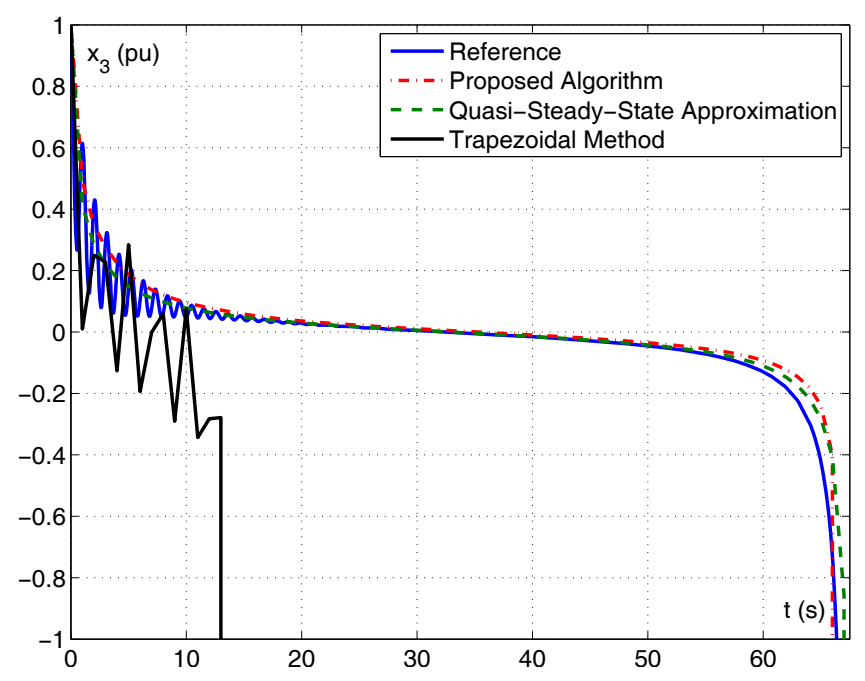

Fig. 2. Small disturbance

The advantage of integration methods with stiff decay lies in their ability to skip rapidly varying solution details while maintaining a decent description of the coarse behavior of the solution. Conversely, integration methods without stiff decay need to be solved with small step size even if only a coarse behavior of the solution is sought, otherwise errors get propagated and pronounced numerical oscillations are experienced [5].

\section{A simple example}

Consider the following system of ODEs:

$$
\begin{aligned}
& \dot{x_{1}}=x_{2} \\
& \dot{x_{2}}=-36 x_{1}-0.5 x_{2}-u(t) \\
& \dot{x_{3}}=M x_{2}-x_{3}^{2}+1-1.002 u(t)
\end{aligned}
$$

where $u(t)$ denotes the unit step function. The initial conditions are $x_{1}(0)=x_{2}(0)=0$ and $x_{3}(0)=1$. The variable of interest is $x_{3}$. When $M=0, x_{3}$ evolves in an unstable aperiodic way, typical of a saddle-node bifurcation. With $M \neq 0$, the comparatively fast oscillation produced by $x_{1}$ and $x_{2}$ is added to that evolution. The disturbance stems from the step function. Its magnitude is adjusted through the value of $M$.

The QSS approximation of the slow dynamics is obtained by considering $x_{1}$ and $x_{2}$ as fast variables, which yields:

$$
\begin{aligned}
0 & =x_{2} \\
0 & =-36 x_{1}-u(t) \\
\dot{x_{3}} & =-x_{3}^{2}+1-1.002 u(t)
\end{aligned}
$$

In this particular example, the QSS approximate model does not depend on the severity of the disturbance, measured by $M$. Hence, even if the disturbance is so severe that it leads to a fast instability, the QSS model will not reveal it.

Figure 2 shows the effect of a "small" disturbance $(M=10)$. The reference response has been obtained with TM using a small time step of $0.001 \mathrm{~s}$. The oscillation period is in the order of $1 \mathrm{~s}$. When using a large time step $h=$

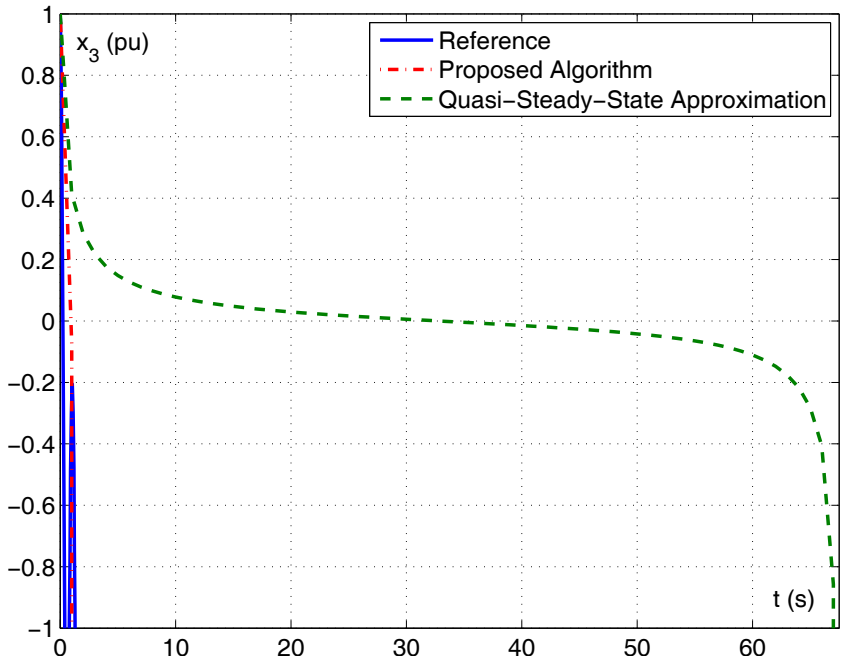

Fig. 3. Large disturbance

$1 \mathrm{~s}$, TM experiences artificial numerical oscillations due to the propagation of errors, while BEM provides a nice coarse approximation of the reference solution. Integrating the QSS model with the same step size gives results very close to the reference and the BEM solution.

Figure 3 shows the effect of a "large" disturbance ( $M=$ 40) leading to a fast instability. Using the same large time step $h=1 \mathrm{~s}$, BEM provides a coarse approximation of the reference solution. Since it is independent of $M$, the QSS solution is the same as in the previous case. Compared to QSS approximation, BEM shows a better ability to track fast aperiodic changes.

\section{Newton iterations}

We consider a simultaneous scheme in which the AEs (1) and the algebraized ODEs stemming from (2) are solved together by Newton method. Traditionally, the other option is to handle the two sets separately, in a partitioned way, while performing functional iterations on $\mathrm{x}$. This combination, however, would not converge when increasing the time step to the extent considered in this work [6].

Assuming algebraized ODEs written in compact form as:

$$
\tilde{\mathbf{f}}(\mathbf{x}, \mathbf{y})=\mathbf{0}
$$

where the dependence on $\mathbf{z}$ is dropped for clarity, the $\nu$-th iteration of the Newton method requires to solve the linear system:

$$
\begin{aligned}
{\left[\begin{array}{cc}
\underset{\mathbf{g}}{\mathbf{g}} & \mathbf{g}_{\mathbf{x}} \\
\tilde{\mathbf{f}}_{\mathbf{y}} & \tilde{\mathbf{f}}_{\mathbf{x}}
\end{array}\right]\left[\begin{array}{c}
\mathbf{y}^{\nu}-\mathbf{y}^{\nu-1} \\
\mathbf{x}^{\nu}-\mathbf{x}^{\nu-1}
\end{array}\right] } & =\left[\begin{array}{c}
-\mathbf{g}\left(\mathbf{x}^{\nu-1}, \mathbf{y}^{\nu-1}\right) \\
-\tilde{\mathbf{f}}\left(\mathbf{x}^{\nu-1}, \mathbf{y}^{\nu-1}\right)
\end{array}\right] \\
& =\left[\begin{array}{l}
\mathbf{b}_{\mathbf{y}}^{\nu-1} \\
\mathbf{b}_{\mathbf{x}}^{\nu-1}
\end{array}\right]
\end{aligned}
$$

where $g_{y}$ is the Jacobian matrix of $g$ with respect to $\mathbf{y}$, and similarly for the other matrices, while $\mathbf{b}_{\mathbf{y}}$ and $\mathbf{b}_{\mathbf{x}}$ are "mismatch" vectors, relative to algebraic and differential equations, respectively. For efficiency reasons, a "dishonest" Newton method is used, in which the Jacobian is updated as rarely as possible [1]. 


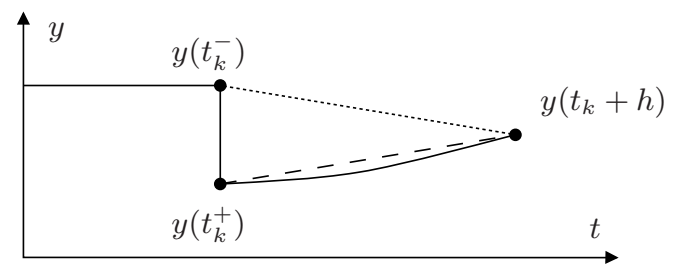

Fig. 4. Solving a discontinuity

At the $k$-th time step, the mismatch vector $\mathbf{b}_{\mathbf{x}}^{\nu-1}$ of TM can be easily obtained as:

$$
\begin{aligned}
\mathbf{b}_{\mathbf{x}}^{\nu-1} & =-\mathbf{c}_{k-1}-\frac{\mathbf{f}\left(\mathbf{x}_{k}^{\nu-1}, \mathbf{y}_{k}^{\nu-1}\right)}{2}+\frac{\mathbf{x}_{k}^{\nu-1}}{h} \\
\text { with } \quad \mathbf{c}_{k-1} & =\frac{\mathbf{x}_{k-1}}{h}+\frac{\mathbf{f}\left(\mathbf{x}_{k-1}, \mathbf{y}_{k-1}\right)}{2}
\end{aligned}
$$

where $\mathbf{c}_{k-1}$ is a constant at all iterations $\nu$ performed at step $k$. A similar derivation for BEM yields:

$$
\begin{aligned}
\mathbf{b}_{\mathbf{x}}^{\nu-1} & =-\mathbf{c}_{k-1}-\mathbf{f}\left(\mathbf{x}_{k}^{\nu-1}, \mathbf{y}_{k}^{\nu-1}\right)+\frac{\mathbf{x}_{k}^{\nu-1}}{h} \\
\text { with } \quad \mathbf{c}_{k-1} & =\frac{\mathbf{x}_{k-1}}{h}
\end{aligned}
$$

\section{E. Discontinuity handling}

If an external disturbance is applied to the system or a discrete transition (3) takes place at time $t_{k}$, the differential variables $\mathbf{x}$ remain unchanged, while the algebraic variables $\mathbf{y}$ undergo a discontinuity, as illustrated in Fig. 4. In principle, a re-initialization is required, in which the new value $\mathbf{y}\left(t_{k}^{+}\right)$ is computed and used in (2) to obtain the new derivative $\dot{\mathbf{x}}\left(t_{k}^{+}\right)$. If so, a reduced system involving $\mathrm{g}_{\mathbf{y}}$ has to be built, factorized and solved. Alternatively, the parameter change can be "stretched" over a very small time step. This also has a cost, since the drastic reduction of the step size $h$ requires to update the Jacobian in (21).

This procedure, however, can be skipped whenever $\mathbf{c}_{k-1}$ does not depend on $\mathbf{y}_{k-1}$, because the mismatch vector does not involve any value that is modified by the discontinuity. In this case, there is no need to solve for $\mathbf{y}\left(t_{k}^{+}\right)$and the simulation may just proceed with the new parameters. The above condition on $\mathbf{c}_{k-1}$ does not hold for TM, as shown by Eq. (22), while it does for BEM, as can be seen from (24).

When computational efficiency is sought, the opportunity of avoiding discontinuity handling - and the resulting Jacobian updates and factorizations - is a key feature. This is a significant advantage in view of the numerous discrete changes that occur in the simulation of power system long-term dynamics, as stressed in the Introduction. In this respect, it is preferable to represent discrete controllers through discrete change in parameters of the type (3) rather than by a continuous-time approximation, which would add rows and columns to the Jacobian matrix.

\section{PROPOSED APPROACH}

We focus on getting simplified solutions of the full model (1-3). In so far as accuracy requirements are relaxed, BEM appears attractive thanks to the possibility it offers to make large time steps without experiencing numerical oscillations, and to pass through discontinuities without re-initializations.

\section{A. Step size control}

Large integration steps are desirable, for computation efficiency reasons, and made possible by the stiff decay property of BEM. However, there are reasons for not taking excessive step sizes:

1) some long-term oscillations of interest (e.g. frequency response in isolated or hydro-generation dominated systems) would be filtered out by large steps;

2) discrete events would be excessively delayed and/or synchronized. To preserve computational efficiency, the exact transition times are not identified (no interpolation is used) but rounded to the next integration step. Hence, too large a step size may delay the transition and artificially synchronize multiple events;

3) when fast transients are triggered, smaller steps may be needed to track the system response and avoid divergence of the Newton iterations.

The proposed approach consists in: (i) integrating with a maximum step $\bar{h}$ chosen in accordance with the first two requirements above, (ii) reducing the step size automatically when the Newton iterations exhibit convergence difficulties, and (iii) recovering to $\bar{h}$ as soon as possible.

Note that this step size control differs from the traditional Local Truncation Error (LTE) control [6]. Indeed, the latter criterion makes little sense in so far as accuracy requirements are relaxed and the emphasis is put on the coarse response of the system.

The logic to control the step size, when convergence difficulties are met (see item 3. above), consists of bounding the mismatch vector $\mathbf{b}_{\mathbf{x}}$ of the first Newton iteration.

Consider again the test equation (11), and assume a linear predictor $^{1}$ (explicit Euler method) is used to obtain a first guess of $x$ at the $k$-th step, i.e.

$$
x_{k}^{0}=x_{k-1}+h \dot{x}_{k-1}=x_{k-1}+h\left(\lambda x_{k-1}\right)
$$

Substituting this expression for $x_{k}^{0}$ in (23) and (24), with $\nu=$ 1, yields:

$$
b_{x}^{0}=-\frac{x_{k-1}}{h}-\lambda x_{k}^{0}+\frac{x_{k}^{0}}{h}=-h \lambda^{2} x_{k-1}
$$

which shows that the mismatch $b_{x}^{0}$ varies linearly with the step size $h$. This property was found to apply pretty well to the mismatch vector of the nonlinear power system models. In this case, the infinite norm $\left\|\mathbf{b}_{\mathbf{x}}^{0}\right\|_{\infty}$ (largest component magnitude) of the first mismatch vector is considered.

Thus, when a convergence problem is detected, the step size is reduced so as to bring $\left\|\mathbf{b}_{\mathbf{x}}^{0}\right\|_{\infty}$ near some value $\tau$. Namely, if a previous step size $h_{k-1}$ brings a mismatch norm:

$$
\left\|\mathbf{b}_{\mathbf{x}}^{0}\right\|_{\infty}=d_{k} h_{k-1}
$$

then, the step size $h_{k}$ that will make the new mismatch norm equal to the bound $\tau$ is given by:

$$
\tau=d_{k} h_{k}
$$

\footnotetext{
${ }^{1}$ since BEM is a first-order integration method, it does not make sense to use more than first-order Taylor expansion for prediction [6]
} 


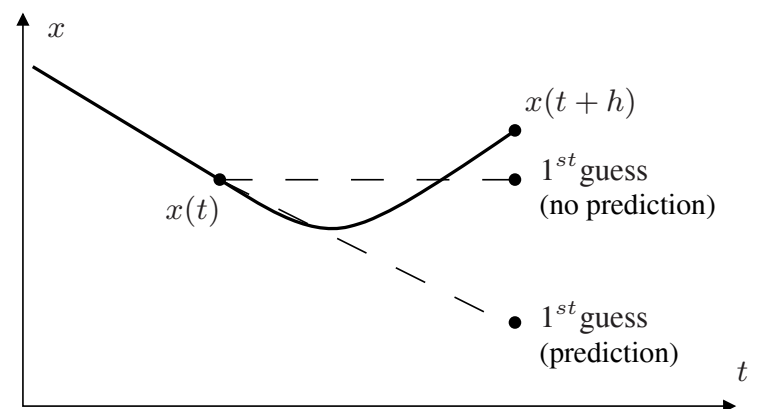

Fig. 5. A case in which prediction is not useful

Dividing (27) by (26) yields:

$$
h_{k}=h_{k-1} \frac{\tau}{\left\|\mathbf{b}_{\mathbf{x}}^{0}\right\|_{\infty}}
$$

As convergence of the Newton sequence improves over successive time steps, the same formula yields increasing values of $h_{k}$. Once the latter reaches $\bar{h}$, the step size is locked to this value until a new convergence problem, if any, calls for a new reduction.

The two parameters of the proposed step size control are thus $\bar{h}$ and $\tau$. Their choice is somewhat system dependent and may require some trial-and-error adjustments, as with any numerical integration procedure.

\section{B. Dealing with prediction}

Prediction is present in many numerical integration methods, either to speed up convergence or as part of the LTE estimation. With the step size control presented in the previous section, prediction was found to be beneficial most of the time but detrimental on some occasions where system state variables evolve rapidly. Indeed, when large steps are made, the predicted value of $\mathbf{x}$ may fall far from the solution point, causing the Newton iterations to diverge. As sketched in Fig. 5, it may be more advantageous not to use prediction in those cases. This was already observed in [1] in the context of detailed time simulation.

The decision to use prediction or not is taken as follows. As the solution is not known when starting the iterations, the initial guess closest to the solution cannot be identified. Instead, the norm $\left\|\mathbf{b}_{\mathbf{x}}^{0}\right\|_{\infty}$ of the mismatch vector is used again. When a first-order prediction is used:

$$
\mathbf{x}_{k}^{0}=\mathbf{x}_{k-1}+h \mathbf{f}\left(\mathbf{x}_{k-1}, \mathbf{y}_{k-1}\right)
$$

and the mismatch vector is given by (24), where $\nu=1$ :

$$
\mathbf{b}_{\mathbf{x}}^{0}=-\frac{\mathbf{x}_{k-1}}{h}-\mathbf{f}\left(\mathbf{x}_{k}^{0}, \mathbf{y}_{k-1}\right)+\frac{\mathbf{x}_{k}^{0}}{h}
$$

while when no prediction is used:

$$
\mathbf{x}_{k}^{0}=\mathbf{x}_{k-1}
$$

and the mismatch vector becomes:

$$
\mathbf{b}_{\mathbf{x}}^{0}=-\frac{\mathbf{x}_{k-1}}{h}-\mathbf{f}\left(\mathbf{x}_{k-1}, \mathbf{y}_{k-1}\right)+\frac{\mathbf{x}_{k-1}}{h}=-\dot{\mathbf{x}}_{k-1}
$$

Thus, with no prediction, the mismatch vector amounts to minus the vector of state variable derivatives at the previous

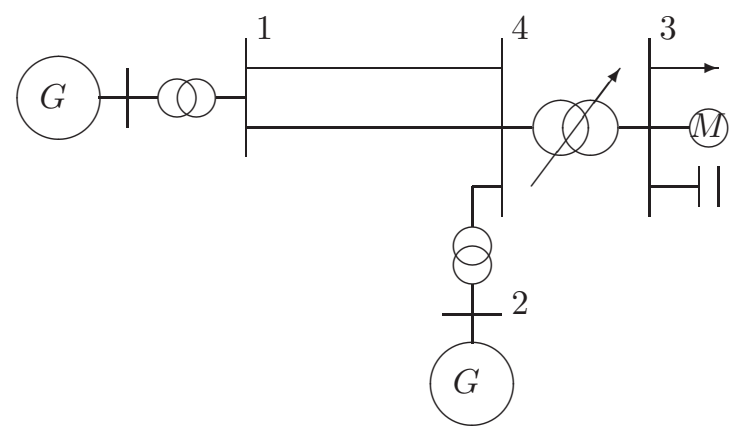

Fig. 6. One-line diagram of the test system

time step. From there on, prediction will be enabled if $\left\|\mathbf{b}_{\mathbf{x}}^{0}\right\|_{\infty}$ computed from (30) is smaller than $\left\|\dot{\mathbf{x}}_{k-1}\right\|_{\infty}$. It should be emphasized that this test comes at the negligible cost of evaluating the time derivatives (if (31) is selected) or at no cost (if (29) is selected).

\section{Application to A SMAll test system}

\section{A. Test system}

We present hereafter simulation results obtained on a 4-bus test system, whose one-line diagram is shown in Fig. 6. This is a variant of the system considered in [4] to illustrate long-term voltage instability mechanisms.

In this system, a large load is fed by an external system through a long double-circuit line, as well as by a local generator controlling the voltage at the close bus 2 . The long-term dynamics comes from the Load Tap Changer (LTC) controlling the voltage of the distribution bus 3, and the OverExcitation Limiter (OEL) protecting the generator at bus 2. The LTC delay is $20 \mathrm{~s}$ on the first tap change and $10 \mathrm{~s}$ on subsequent changes. The OEL has an inverse-time characteristic [4].

With respect to the model detailed in [4], the Thévenin equivalent at bus 1 has been replaced by a large equivalent generator behind its step-up transformer, while both generators are driven by a steam turbine with speed governor. The purpose of this modification is to have the slower, common frequency mode of oscillation in the system responses.

The disturbance considered is the tripping of one of the two circuits, at $t=1 \mathrm{~s}$. This outage, together with the reactive power limitation of the generator at bus 2 , causes the maximum power deliverable to the load to decrease below the level that the LTC tries to restore indirectly. Hence, long-term voltage instability results.

All phasors are projected on reference axes rotating at the speed of the Center Of Inertia (COI) and rotor angles are defined with respect to this COI reference. This is essential to avoid phasors to rotate and rotor angles to increase with time, when the system settles at a new frequency. This may cause divergence of Newton iterations when making large steps, or at least it may require to update the Jacobian frequently.

\section{B. Simulation methods}

The objective is to compare the time responses provided by respectively: 


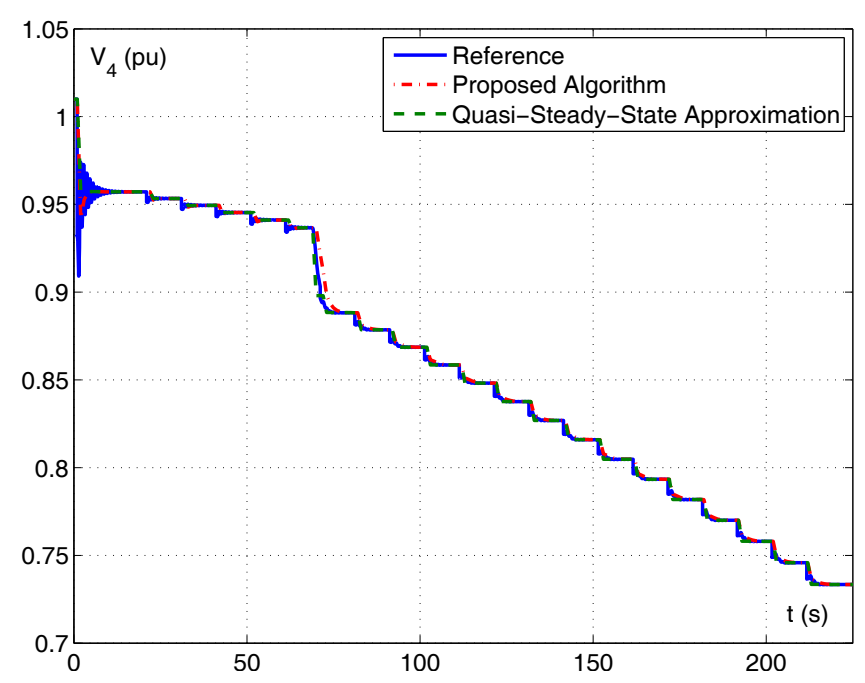

Fig. 7. Case 1. Transmission side voltage

1) a reference method. This is full simulation of the full model (1-3). For accuracy purposes, TM has been used with variable step size controlled by LTE;

2 ) the proposed algorithm. The default step size $\bar{h}$ has been taken as $1 \mathrm{~s}$. The mismatches are monitored over successive iterations to early detect possible divergence. In the latter case, the iterations are stopped, and the step size $h$ is reduced as described in Section III-A;

3 ) the QSS approximation of the type (4-7), using a constant step size $h=1 \mathrm{~s}$. The retained dynamics (6) relate to the non-windup integrator that determines the instant of OEL activation as well as the steam turbine reheat time constant. These ODEs have been integrated by BEM.

The relevant Jacobian matrices are evaluated numerically, using finite differences. They are updated at the beginning of an iteration if the decrease of the mismatches is not large enough. An update also takes place in case a state variable reaches or leaves a limit.

\section{Case 1: "pure" long-term voltage instability}

In this first scenario, the whole load is represented with an exponential model. The time evolutions of the voltage at bus 4 computed by the three methods are shown in Fig. 7. The electromechanical oscillations that follow the line tripping die out rather fast, indicating that the short-term dynamics are stable. The LTC starts responding at $t=21 \mathrm{~s}$, while the OEL is activated at $t \simeq 70 \mathrm{~s}$. From there on, the LTC fails restoring the distribution voltage and depresses the transmission one. This degradation stops when the LTC hits its limit.

Both the proposed approach and the QSS approximation provide evolutions almost undistinguishable from the benchmark method.

\section{Case 2: long-term instability causing loss of synchronism}

This scenario differs from Case 1 by a larger active power generation at bus 2 . Figure 8 shows that the voltage starts evolving as in the previous case, with an OEL activation at

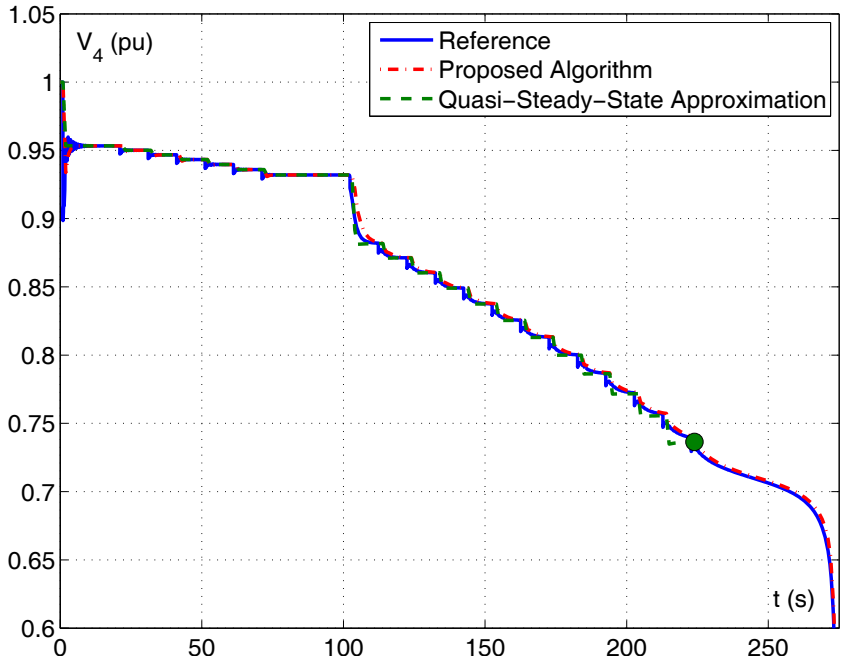

Fig. 8. Case 2. Transmission side voltage

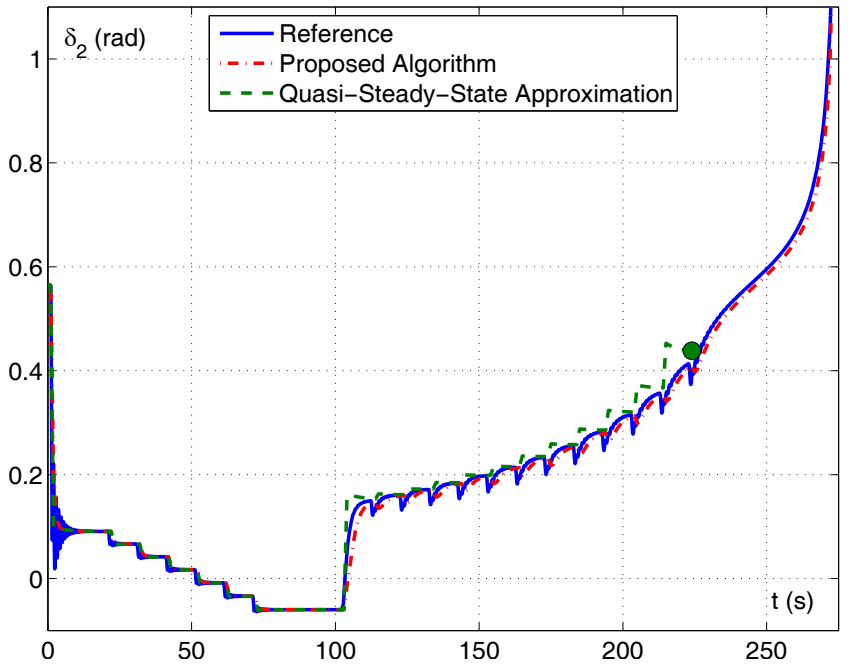

Fig. 9. Case 2. Rotor angle of generator at bus 2

$t \simeq 105 \mathrm{~s}$, but eventually plunges down at about $t \simeq 274 \mathrm{~s}$. This is caused by a loss of synchronism, as seen from the rotor angle in Fig. 9 (generator at bus 2, referred to COI).

The proposed method provides a system evolution hardly distinguishable from the benchmark, thereby showing its ability to track fast aperiodic changes. The QSS simulation, on the other hand, reaches a singularity at $t=224 \mathrm{~s}$, identified by dots in Figs. 8 and 9. Although long-term voltage instability can be diagnosed from the QSS response, the resulting fast instability cannot be identified by this method.

\section{E. Case 3: long-term instability causing motor stalling}

This scenario differs from Case 1 by the load model. Part of the load consists of an equivalent induction motor supposed to represent multiple motor loads.

Figure 10 shows that the voltage evolves under the effect of rotor oscillations, two tap changes, and the OEL activation at $t \simeq 35 \mathrm{~s}$. Immediately after, it falls sharply. As can be seen from the slip curve in Fig. 11, this is caused by the stalling of the motor (which decelerates up to standstill; no tripping 


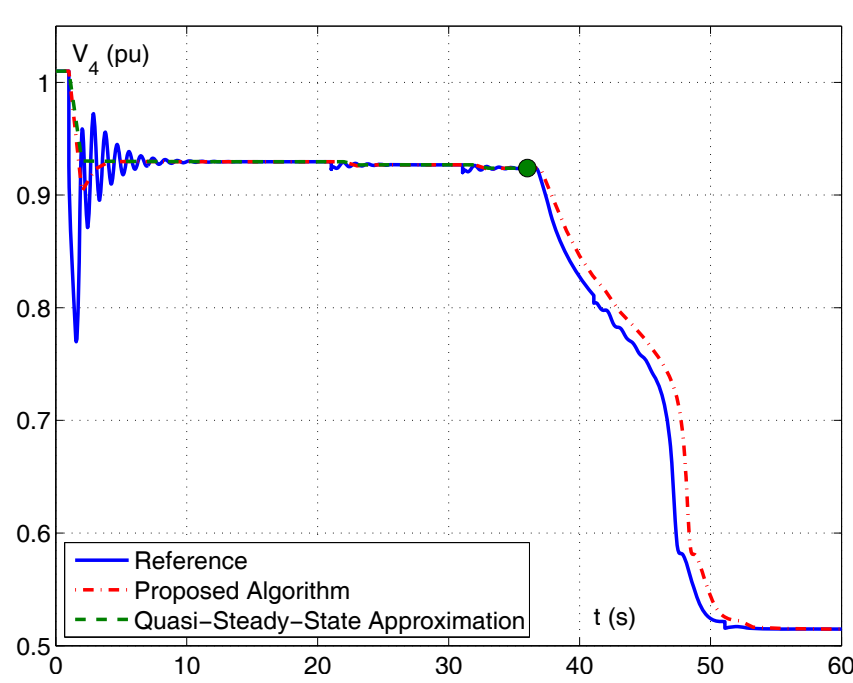

Fig. 10. Case 3. Transmission side voltage

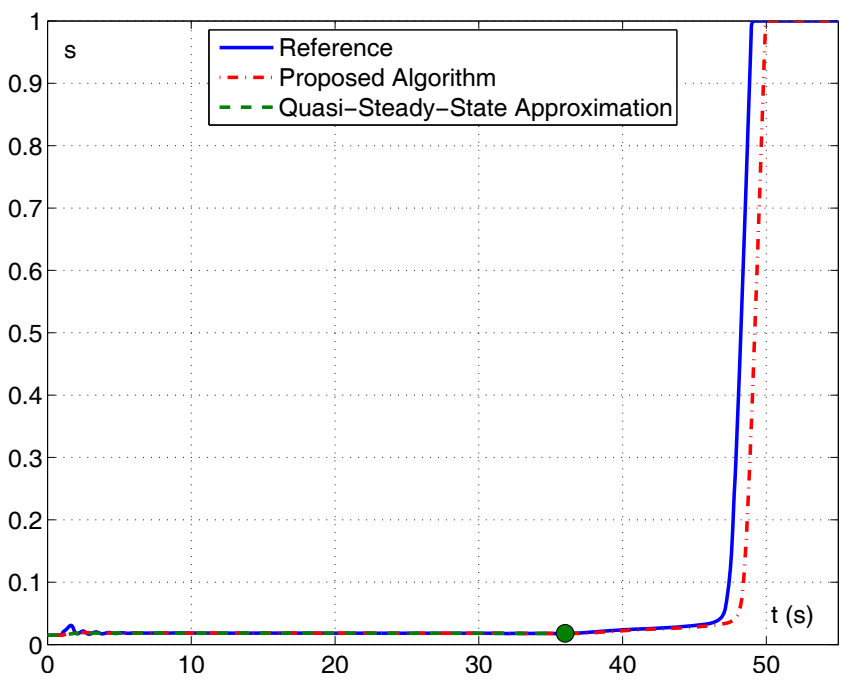

Fig. 11. Case 3. Motor slip

by a protection has been assumed), which itself results from the lack of voltage support and reactive power supply after the OEL activation.

Once again, the proposed method provides a response quite close to the benchmark, and shows its ability to track fast aperiodic changes. The QSS simulation, on the other hand, reaches a singularity, identified by dots in Figs. 10 and 11. This takes place at $t=36 \mathrm{~s}$, when the field current limit is enforced, and at a significantly higher voltage than in Case 2 . Complementary analysis of that singularity is needed for a satisfactory diagnosis.

\section{F. Step size control in proposed method}

Figure 12 shows the evolution of the step size in Case 3. Up to $t=46 \mathrm{~s}$, the default step size $\bar{h}=1 \mathrm{~s}$ has been successfully used. At $t=46 \mathrm{~s}$, convergence difficulties are detected, most likely due to the fast change in motor slip (see Fig. 11). Hence, the step starts being adjusted according to Eq. (28), where $\tau$ has been set to 2 . At $t \simeq 52 \mathrm{~s}, h$ is set back to $\bar{h}$ for the remaining of the simulation.

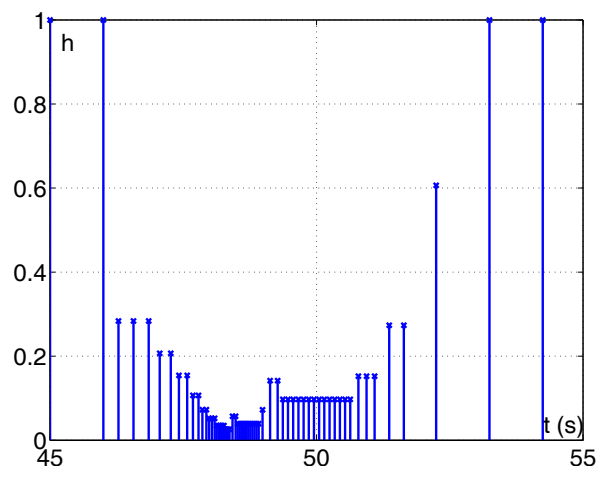

Fig. 12. Case 3. Evolution of step size $h$ for $45 \leq t \leq 55 \mathrm{~s}$

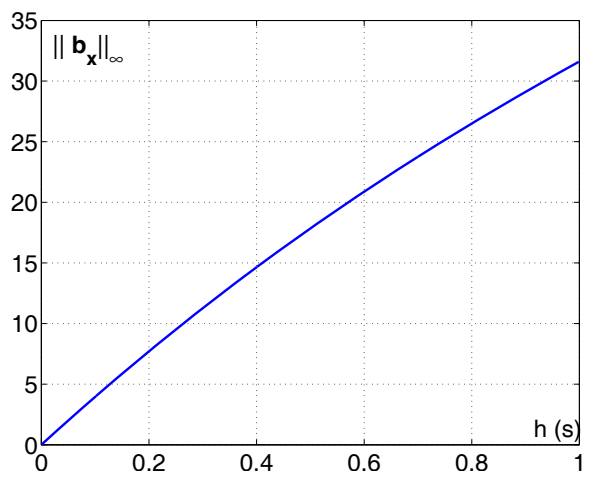

Fig. 13. Example of variation of $\left\|\mathbf{b}_{\mathbf{x}}^{0}\right\|_{\infty}$ with the step size $h$

TABLE I

COMPUTATIONAL EFFORT OF VARIOUS METHODS

\begin{tabular}{|c|c|rr|rr|rr|}
\hline Case & simulated & \multicolumn{2}{|c|}{ TM with } & \multicolumn{2}{c|}{ proposed } & \multicolumn{2}{c|}{ QSS } \\
No & time (s) & \multicolumn{2}{|c|}{ LTE control } & \multicolumn{2}{c|}{ method } & \multicolumn{2}{c|}{ simulation } \\
& & JU & NI & JU & NI & JU & NI \\
\hline 1 & 300 & 291 & 8115 & 27 & 440 & 19 & 298 \\
2 & 224 & 287 & 8526 & 22 & 494 & 22 & 322 \\
2 & 274 & 295 & 10241 & 34 & 874 & final divergence \\
3 & 36 & 76 & 1580 & 8 & 59 & 9 & 57 \\
3 & 300 & 272 & 6777 & 54 & 652 & final divergence \\
\hline
\end{tabular}

The variation of $\left\|\mathbf{b}_{\mathbf{x}}^{0}\right\|_{\infty}$ with the step size $h$ is shown in Fig. 13. The latter relates to the first iteration of the first time step after the line is tripped, in Case 1 , at $t=1 \mathrm{~s}$. The variation is not far from linear, as for the test equation (11). This holds true even for relatively large steps.

\section{G. Computational efforts}

Table I compares the computational effort of respectively the reference, the proposed and the QSS method in the three cases presented so far, and for different simulation horizons. The numbers of Newton iterations ("NI" in the table) and Jacobian updates ("JU" in the table) are provided as measures of this effort. The size of the Jacobian is the same for the three methods.

As can be seen, the proposed method is much less demanding than TM. It even approaches the high computational efficiency of the QSS approach. The additional effort with respect to the latter is largely spent in computing the fast changes that the QSS approximation cannot reproduce. 


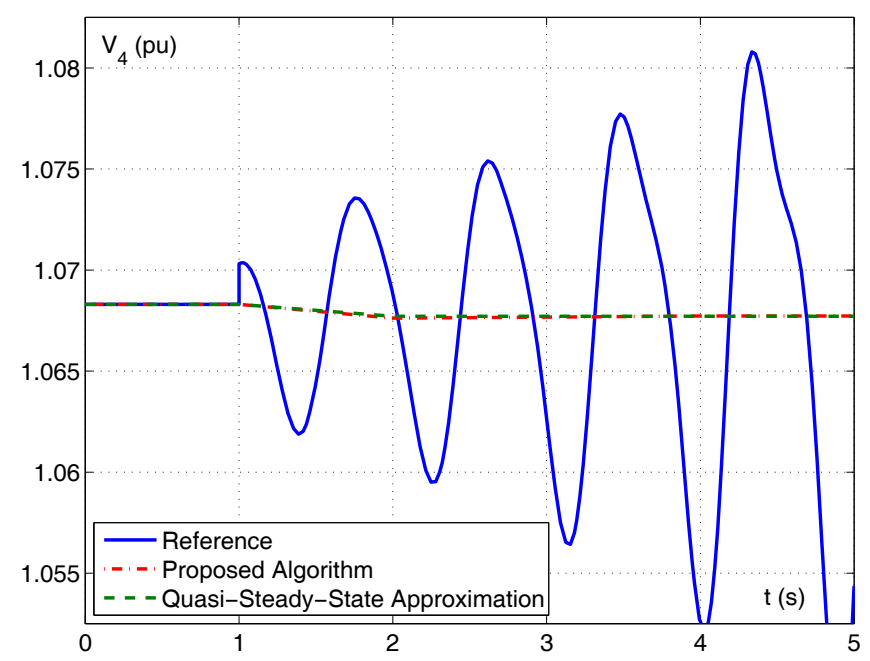

Fig. 14. Case 4. Transmission side voltage

\section{H. Case 4: oscillatory instability}

The last case illustrates the hyper-stability drawback of BEM, already mentioned in Section II-A. The gain of the AVR of the generator at bus 2 has been increased so that after the line outage the generator lacks damping torque. The resulting oscillatory unstable response is shown with solid line in Fig. 14. However, both the QSS approximation and BEM ignore this unstable oscillation, and provide a trajectory which is close to the underlying unstable equilibrium of the system.

Seeking an analogy with the test equation (11), one can say that the simulation corresponds to point B in Fig. 1, where the numerical response is stable but the real response is not. The $\mathrm{TM}$, on the other hand, would show an unstable response.

Several remedies could be thought of [9]:

1) escape the BEM stability region by reducing the step size, which corresponds to moving from point $\mathrm{B}$ to point $\mathrm{C}$ in Fig. 1. Coming back to the proposed approach, it would be required to reduce the value of $\bar{h}$. Alternatively, the step size could be controlled by Eq. (28) with a significantly lower value of $\tau$;

2) switch to a higher-order stiff-decay integration method, less prone to hyper-stability, and preserving the interesting features of BEM;

3) switch to another solver, such as TM, not prone to hyperstability.

The change in simulation scheme outlined could be made over limited periods of time. However, since the proposed method does not have an indication of emerging oscillatory instability, it should take place somewhat systematically after potentially dangerous events such as faults, line or generator outages, etc.

Of course, in terms of computational effort the above remedies will affect the overall performance of the proposed approach and may bring it close to a non-simplified simulation.

\section{CONClusion}

On the premise that computational efficiency may be more important than high accuracy for some applications, this paper demonstrates the possibility to perform simplified but fast time-domain simulations of a detailed dynamic model of the power system. Numerical approximation of the system response is thus preferred to simplification of the model, typical of the QSS approximation.

The proposed integration scheme allows neglecting the oscillatory dynamics which are fast compared to the step size (such as inter-machine rotor oscillations) while concentrating on the average evolution, with the possibility to track fast changes such as loss of synchronism and motor stalling. This is obtained by exploiting the stiff decay property of the backward Euler method, which also allows a simplified handling of discontinuities.

A variable step strategy has been proposed which consists of integrating with a maximum step unless convergence difficulties of the Newton iterations are met, corresponding to fast changes. Then, the strategy focuses on the magnitude of the Newton mismatches, until the step size can be brought back to its large default value.

Oscillatory instability is likely to remain outside the range of phenomena that can be simulated by the proposed approach. Systems with lightly damped electromechanical modes should thus be considered with care. This is part of the compromise between accuracy and computational efficiency.

Future work will be devoted to: (i) detection of emerging oscillatory instability (e.g. through tracking of dominant system eigenvalues), (ii) alternative stiff-decay integration schemes, (iii) large-scale tests to assess the gain in computing times.

\section{REFERENCES}

[1] B. Dembart, A.M. Eirsman, E.G. Cate, M.A. Epton and H. Dommel, "Power System Dynamic Analysis - Phase I. Final Report EPRI EL-484", July 1977

[2] B. Stott, "Power systems dynamic response calculations", Proc. of the IEEE, Vol. 67, Feb. 1979

[3] P. Kundur, Power System Stability and Control, Mc Graw Hill, EPRI Power System Engineering Series, 1994

[4] T. Van Cutsem, C. Vournas, Voltage Stability of Electric Power Systems, Springer (previously Kluwer Academic Publishers), 1998

[5] U.M. Ascher, L.R. Petzold, Computer Methods for Ordinary Differential Equations and Differential-Algebraic Equations, Society for Industrial and Applied Mathematics (SIAM), 1998

[6] C.W. Gear, Numerical Initial Value Problems in Ordinary Differential Equations, Prentice-Hall, 1971

[7] H.W. Dommel, N. Sato, "Fast Transient Stability Solutions", IEEE Trans. on Power Apparatus and Systems, Vol. 91, July-Aug. 1972

[8] R.B.I. Johnson, B.J. Cory, M.J. Short, "A tunable integration method for the simulation of power system dynamics", IEEE Trans. on Power Systems, Vol. 3, No. 4, Nov. 1988

[9] J.Y. Astic, A. Bihain, M. Jerosolimski, "The mixed Adams - BDF variable step size algorithm to simulate transient and long term phenomena in power systems", IEEE Trans. on Power Systems, Vol. 9, No. 2, May 1994

[10] J.J. Sanchez-Gasca, R. D’Aquila, W.W. Price, J.J. Paserba, "Variable time step, implicit integration for extended-term power system dynamic simulation", Proc. 1995 Power Industry Computer Application (PICA) conf., pp. 7-12, May 1995

[11] A. P. Meliopoulos, G. J. Cokkinides, G. K. Stefopoulos, "Quadratic Integration Method", Proc. 2005 International Power System Transients (IPST) conf., Montreal (Canada), 19-23 June 2005

[12] M.-E. Grenier, D. Lefebvre, T. Van Cutsem, "Quasi steady-state models for long-term voltage and frequency dynamics simulation", Proc. IEEE Power Tech conference, St Petersburg (Russia), June 2005

[13] T. Van Cutsem, M.-E. Grenier, D. Lefebvre, "Combined detailed and quasi steady-state time simulations for large-disturbance analysis", Electrical Power and Energy Systems, Vol. 28, 2006, pp. 634-642 